\title{
Effects of amiodarone on thyroid function
}

\author{
Shaminda Kahandawa ${ }^{1}$, Noel Somasundaram ${ }^{2}$ \\ Sri Lanka Journal of Diabetes Endocrinology and Metabolism 2011; 1: 25-27
}

\section{Introduction}

Amiodarone is an effective drug used in the management of ventricular and resistant supraventricular tachyarrhythmias. But it has multiple effects on the thyroid which include abnormalities of thyroid function tests, hypothyroidism and thyrotoxicosis. In Sri Lanka amiodarone is a widely used antiarrhythmic, in controlling resistant arrhythmias and useful in heart failure. The unavailability of other antiarrhythmics and the high cost of internal cardiac defibrillators (ICD) have favored the use of amiodarone. However, the relationship between amiodarone therapy and thyroid dysfunction is not adequately recognized and evaluated in day to day practice.

\section{Pharmacology of amiodarone}

The structural formula of amiodarone closely resembles that of thyroid hormones (1). Amiodarone is a benzofuran derivative and iodine is responsible for $37 \%$ of its weight. Therefore at a standard dose of $100-600 \mathrm{mg}$ per day, recipients are exposed to $3-21 \mathrm{mg}$ iodine per day, which is over 35-140 times the recommended daily allowance of iodide (recommended dose $=150 \mu \mathrm{g}$ per day) (2). The average half-life of amiodarone is 40 days, leading to a long period of effect after drug discontinuation (2).

\section{Clinical use of amiodarone}

Amiodarone is a class III antiarrhythmic, according to the Vaughan-Williams classification. Its mechanism of action is inhibition of myocardial $\mathrm{Na}^{+} \mathrm{K}^{+}$-ATPase activity (1). Amiodarone is indicated for the treatment of lifethreatening recurrent ventricular arrhythmias and in controlling resistant supraventricular tachyarrythmias. The ability to use amiodarone in heart failure is an additional advantage.

\section{Effects of amiodarone on thyroid function}

Abnormalities of thyroid function tests may occur with amiodarone therapy in otherwise clinically euthyroid patients. In the peripheral tissues, particularly liver, amiodarone inhibits conversion of $\mathrm{T}_{4}$ to $\mathrm{T}_{3}$. In addition, the drug inhibits $\mathrm{T}_{4}$ entry into peripheral tissues (2). Both mechanisms lead to increased serum $\mathrm{T}_{4}$ and decreased serum $T_{3}$ concentrations in euthyroid subjects who are on amiodarone therapy. Also it increases the concentration of reverse $\mathrm{T}_{3}$. Amiodarone may increase serum TSH concentration during the early months of treatment (2). These changes are believed to be related to decrease in intracellular $\mathrm{T}_{4}$ entry and inhibition of $\mathrm{T}_{4}$ to $\mathrm{T}_{3}$ conversion in the pituitary gland. With long term amiodarone treatment ( $>3$ months) TSH levels will normalize, while free $\mathrm{T}_{4}$ and reverse $\mathrm{T}_{3}$ may be slightly elevated.

\section{Amiodarone-induced hypothyroidism (AIH)}

Most patients treated with amiodarone will remain euthyroid throughout the treatment course. However, $10-20 \%$ of patients treated short term will manifest AIH (3), slightly more frequent in females, with a female to male ratio of 1.5:1. This occurs more frequently in iodinesufficient areas, where AIH usually develops in patients with underlying Hashimoto thyroiditis.

The most likely pathogenic mechanism is that the thyroid gland already damaged by preexisting Hashimoto thyroiditis is unable to escape from the acute WolffChaikoff effect after an iodine load and fails to resume normal thyroid hormone synthesis (2). Alternatively, amiodarone may accelerate the natural course of Hashimoto thyroiditis via iodine-induced damage to the thyroid follicles. The clinical manifestations and findings of thyroid function tests are similar to those of primary hypothyroidism. If hypothyroidism is sustained or severe, AIH may precipitate ventricular arrhythmias such as torsades de pointes (1).

Treatment: If amiodarone is needed for the underlying cardiac disorder, it can be continued in association with levothyroxine replacement. Because these patients often have severe underlying cardiac disease, it is advisable to maintain the serum TSH concentration in the upper half of the normal range (2). Since amiodarone inhibits $\mathrm{T}_{4}$ conversion to $\mathrm{T}_{3}$, larger doses of levothyroxine may be required. Spontaneous remission of hypothyroidism may occur, particularly in patients without underlying Hashimoto thyroiditis.

\section{Amiodarone-induced thyrotoxicosis (AIT)}

The incidence of AIT is reported as $5-10 \%$ in most studies (1). It is relatively more frequent in iodine-deficient areas and particularly in men (male to female incidence

${ }^{1}$ Senior Registrar in Endocrinology, ${ }^{2}$ Consultant Endocrinologist, National Hospital of Sri Lanka. 
ratio is 3:1). AIT may develop early during amiodarone treatment or even several months after drug withdrawal due to its long half life.

Pathogenesis: Type 1 AIT results from the Jod-Basedow effect and is typically seen in patients with preexisting thyroid gland abnormalities (4). Type 2 AIT results from destructive inflammatory thyroiditis caused by amiodarone itself and its high iodine content. Type 2 AIT occurs in patients with otherwise clinically normal thyroid glands at presentation (1). Recent data show that type 2 AIT is by far the most frequent form (4). However, the two mechanisms may coexist in the same patient (mixed or indefinite AIT).

Clinical manifestations: The reappearance or exacerbation of the underlying cardiac arrhythmia, in a patient with previously stable cardiac status while on amiodarone, should prompt an investigation into thyroid function (2). Hyperthyroidism results in potentiation of warfarin effects by increasing the rate of degradation of clotting factors. Therefore hyperthyroidism should also be suspected in a patient with an unexplained decrement in warfarin dosage (2). Classical symptoms of thyro-toxicosis may be absent, due to the antiadrenergic action of amiodarone and its inhibition of conversion of $\mathrm{T}_{4}$ to $\mathrm{T}_{3}$.

Diagnosis: Decreased serum TSH levels and increased serum $\mathrm{T}_{3}$ concentration confirm the diagnosis of AIH. Serum concentrations of $\mathrm{T}_{4}$ may be a less useful indicator of hyperthyroidism than those of $\mathrm{T}_{3}$, because transient increase in $T_{4}$ concentration often takes place after the initiation of amiodarone treatment without hyperthyroidism being present (5).

Identification of the different subtypes of AIT is crucial because this helps determine the best therapeutic approach. Type 1 AIT is suggested by the presence of thyroid abnormalities, such as goiter and positive thyroid autoantibodies. Although not specific, the levels of interleukin 6 (IL-6) are usually elevated in type 2 AIT. Thyroid ultrasonography with colour Doppler studies is a very important diagnostic tool. Most cases of type 2 AIT are characterized by absent hypervascularity, while type 1 AIT usually shows a normal or increased vascularity.

Thyroidal ${ }^{131}$ I uptake (RAIU) can also be used to differentiate the types of AIT. RAIU is usually very low $(<3 \%)$ in type 2 AIT and low-normal, normal, or even increased (despite the iodine load) in type 1 AIT (6).

Management: Since AIT is associated with increased mortality, euthyroidism must be achieved as quickly as possible. On the other hand, the diagnostic uncertainties regarding the type of AIT may lead to treatment with both thionamides and steroids. This approach has a higher risk of drug side effects.

Type 1 AIT is best treated with antithyroid drugs. However, an iodine-replete thyroid gland is less responsive to the inhibitory action of thionamides. Thus, higher drug dosages for longer periods are required to achieve euthyroidism (7). Potassium perchlorate (1g/day for 4-6 weeks), which decreases thyroid iodine uptake may be added to increase the responsiveness. Unfortunately, long term use of potassium perchlorate is limited by its toxic effects such as agranulocytosis and aplastic anemia. Radioactive iodine treatment may be indicated, if RAI uptake values are sufficiently high $(>10 \%)$ after the patient becomes euthyroid (2). Total thyroidectomy is indicated in patients who are resistant to medical therapy and with severe cardiac complications (8).

Type 2 AIT may resolve spontaneously. If indicted, the treatment of choice is steroids. Initial prednisone dose is about $0.5-0.7 \mathrm{mg} / \mathrm{kg} /$ day and the treatment is usually continued for 1 to 3 months (2). Side effects of prednisolone such as fluid retention which can precipitate cardiac failure must be anticipated and treated accordingly. About $50 \%$ of patients become euthyroid within 4 weeks of treatment. Periodic assessment of thyroid function is indicated in these patients, because of the occurrence of hypothyroidism (up to 17\%) during longterm follow up (4).

The most difficult challenge is mixed/indefinite forms of AIT. Treatment of these forms is based on the concomitant use of thionamides (with or without potassium perchlorate) and glucocorticoids (7).

\section{Withdrawal of amiodarone in AIT}

Several facts should be taken into consideration before the withdrawal of amiodarone. The biological effects of amiodarone persist long after cessation of treatment. Therefore drug withdrawal might not influence immediate management. On the other hand, amiodarone may protect the heart from the localized effects of thyrotoxicosis due to its antiadrenergic action and related decreased conversion of $\mathrm{T}_{4}$ to $\mathrm{T}_{3}$. Thus, discontinuation of the drug may actually worsen thyrotoxic effects on the heart (9). Therefore amiodarone withdrawal must be done carefully even if permissible and should be in concurrence with the cardiologist.

\section{Dronedarone}

Dronedarone was approved by the FDA in 2009 for the treatment of atrial fibrillation (1). This drug has similar electrophysiologic properties to those of amiodarone (10), but does not contain iodine. The DIONYSOS study demonstrated that there was significantly lower incidence of thyroid dysfunction in patients treated with dronedarone in comparison to the amiodarone group (1). In the future dronedarone may become an attractive and effective alternative to amiodarone. 


\section{Summary}

Amiodarone is an iodine rich drug commonly used in the treatment of many cardiac tachyarrhythmias. But it has multiple effects on the thyroid which include abnormalities of thyroid function tests, hypothyroidism and thyrotoxicosis. Although the widely used antiarrythmic in Sri Lanka, the clinical impact of these effects are yet to be evaluated. Amiodarone transiently produces alterations in thyroid function tests in euthyroid patients. Understanding these changes is crucial in avoiding unnecessary investigations and treatment. In contrast to amiodarone-induced hypothyroidism, amiodarone-induced thyrotoxicosis is a difficult condition to diagnose and treat. Dronedarone will be the suitable alternative to treat patients with atrial fibrillation who are at risk of developing amiodarone-induced thyroid dysfunction.

\section{References}

1. Cohen-Lehman J, Dahl P, Danzi S, Klein I. Effects of amiodarone therapy on thyroid function. Nat Rev Endocrinol 2010; 6: 34-41.

2. Martino E, Bartalena L, Bogazzi F, Braverman LE. The effects of amiodarone on the thyroid. Endocr Rev 2001; 22: 240-54.
3. Basaria S. Cooper DS. Amiodarone and the thyroid. Am J Med 2005; 118: 706-14.

4. Bogazzi F, et al. Proportion of type 1 and type 2 amiodaroneinduced thyrotoxicosis has changed over a 27 -year period in Italy. Clin Endocrinol 2007; 67: 533-7.

5. Han TS, Williams GR, Vanderpump MPJ. Benzofuran derivatives and the thyroid. Clin Endocrinol 2009; 70: $2-13$.

6. Eskes SA, Wiersinga WM. Amiodarone and thyroid. Best Pract Res Clin Endocrinol Metab 2009; 23: 735-51.

7. Bogazzi F, Bartalena L, Martino E. Approach to the patient with amiodarone-induced thyrotoxicosis. Clin Endocrinol Metab 2010; 95: 2529-35.

8. Gough J, Gough IR. Total thyroidectomy for amiodaroneassociated thyrotoxicosis in patients with severe cardiac disease. World J Surg 2006; 30: 1957-61.

9. Bartalena L, Bogazzi F, Martino E. Amiodarone-induced thyrotoxicosis: a difficult diagnostic and therapeutic challenge. Clin Endocrinol 2002; 56: 23-4.

10. Hohnloser SH, et al. Effect of dronedarone on cardiovascular events in atrial fibrillation. $N$ Engl J Med. 2009; 360: 668-78. 